\title{
INFRARED SPECTRAL ENERGY DISTRIBUTION OF GALAXIES IN THE AKARI ALL SKY SURVEY: CORRELATIONS WITH GALAXY PROPERTIES AND THEIR PHYSICAL ORIGIN
}

\author{
R. Makiya ${ }^{1}$, T. Totani ${ }^{1}$, T. T. Takeuchi ${ }^{2}$, M. Nagashima ${ }^{3}$, and M. A. R. Kobayashi ${ }^{4}$ \\ ${ }^{1}$ Department of Astronomy, School of Science, Kyoto University, Sakyo-ku, Kyoto 606-8502 \\ ${ }^{2}$ Institute for Advanced Research, Nagoya University, Furo-cho, Chikusa-ku, Nagoya 464-8601 \\ ${ }^{3}$ Faculty of Education, Nagasaki University, Nagasaki, Nagasaki 852-8521 \\ ${ }^{4}$ Optical and Infrared Astronomy Division, National Astronomical Observatory, Mitaka, Tokyo 181-8588 \\ E-mail: makiya@kusastro.kyoto-u.ac.jp \\ (Received July 03, 2012; Accepted August 22,2012)
}

\begin{abstract}
We will report our recent study on the properties of more than 1,600 galaxies detected by the AKARI All-Sky Survey with physical quantities based on optical and 21-cm observations, to understand the physics determining the infrared spectral energy distribution (Totani et al., 2011). We discover a tight linear correlation for normal star-forming galaxies between the radiation field strength of dust heating (corresponding to dust temperature) and the galactic-scale infrared radiation field, $L_{\mathrm{TIR}} / R^{2}$. This is the tightest correlation of dust temperature ever known, and the dispersion along the mean relation is $13 \%$ in dust temperature. This relation can be explained physically by a thin layer of heating sources embedded in a thicker, optically-thick dust screen. We also find that the number of galaxies sharply drops when galaxies become optically thin against dust-heating radiation, indicating that a feedback process to galaxy formation (e.g., by the photoelectric heating) is working when dust-heating radiation is not self-shielded on a galactic scale. We discuss implications from these findings for the $M_{\mathrm{H}_{\mathrm{I}}}$-size relation, the Kennicutt-Schmidt relation, and galaxy formation in the cosmological context.
\end{abstract}

Key words: infrared: telescope; conferences: proceedings

\section{INTRODUCTION}

Dust grains in the interstellar medium (ISM) absorb ultraviolet and optical photons emitted from stars, and re-emitted it in infrared and submillimeter wavelengths. The intensity of the cosmic infrared background radiation indicates that about half of the energy originally radiated from stars is eventually reemitted from dust grains (Hauser \& Dwek, 2001). Heavily obscured star formation activity is difficult to detect by observations in the ultraviolet/optical, and infrared/submillimeter observations should be crucially important to get the full picture of the formation and evolution of galaxies. In the last decade, our observational knowledge of the hidden side of galaxy evolution has significantly improved thanks to the advanced infrared/submillimeter facilities such as JCMT/SCUBA, Spitzer, AKARI, and Herschel. Moreover, the Atacama Large Millimeter/submillimeter Array (ALMA) will revolutionize our understanding of galaxy evolution in the near future. In order to extract useful information from these data, we need good theoretical understanding and models of galactic dust emission to be compared.

However, it is not straightforward to predict global (i.e., galactic-scale) SEDs of dust emission for a theoretical model galaxy, in contrast to direct stellar emission that can be calculated by the method of stellar population synthesis. This is because the physics determining 


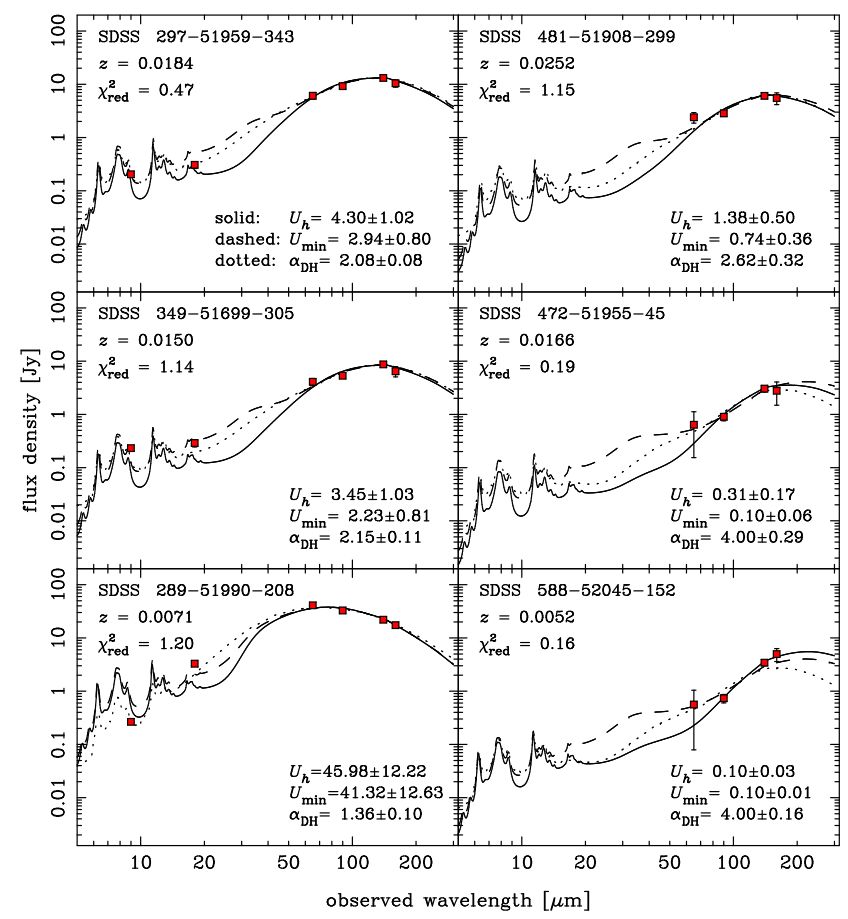

Fig. 1. Examples of the SED fittings to the galaxies in the AKARI-SDSS sample. The squares are AKARI data points. The solid line represents the DL07 model with the parameter $U_{h}$. The unique identification set of plate number, MJD, and fiber number of the SDSS spectroscopic database is also shown in each panel. The dotted and dashes lines represent other SED models than DL07 model with the parameters $U_{\min }$ and $\alpha_{\mathrm{DH}}$. Please see Totani et al. (2011) for more details.

the global SED of dust emission and its relations to other physical properties have remained highly elusive (see Walcher et al., 2011 for a review). An approach often taken in the literature (e.g., Guiderdoni et al., 1998; Takeuchi et al., 2001; Chary \& Elbaz, 2001; Lagache, Dole, \& Puget, 2003; Valiante et al., 2009) is to relate the SED parameters (e.g., the dust temperature $T_{d}$ ) to the total infrared luminosity, $L_{\mathrm{TIR}}$ (defined as the bolometric luminosity of dust emission), based on the observed correlation between the two (higher $T_{d}$ for larger $L_{\mathrm{TIR}}$; Soifer et al., 1987a; Soifer \& Neugebauer, 1991; Chapin et al., 2009). However, there is a large scatter along the mean $L_{\mathrm{TIR}}-T_{d}$ relation (see, e.g., Hwang et al., 2010 for recent data), and it is physically unreasonable to relate an extensive quantity $L_{\mathrm{TIR}}$ that scales with the system size to an intensive quantity $T_{d}$ that does not (Totani \& Takeuchi, 2002). Extension of this relation including another parameter has also been discussed, e.g., galaxy size (Devereux, 1987; Phillips \& Disney, 1988; Lehnert \& Heckman, 1996; Chanial et al., 2007; Rujopakarn et al., 2011a, b), but a consistent physical picture has not yet been established.

In this work we perform SED fittings of physical dust models to the AKARI data of more than 1,600 lowredshift $(0.005 \lesssim z \lesssim 0.1$, the mean redshift is $z \sim 0.03)$ galaxies, and compare the SED parameters with other physical properties of galaxies obtained by optical and 21-cm observations. The goal of this paper is to find the key physical quantities and laws to determine the global SEDs of dust emission (especially around the thermal peak), and give physical interpretations to them.

\section{SAMPLE SELECTION}

We use the AKARI/IRC All-Sky Survey Point Source Catalog (PSC, ver. 1.0) and the AKARI/FIS All-Sky Survey Bright Source Catalog (BSC, ver. 1.0), which were released in March 2010 including 870,973 and 427,071 sources, respectively.

We construct the following three samples by crossmatching the AKARI catalogs with the existing catalogs of nearby galaxies selected in other wavelengths. The first sample is the cross-match with the galaxies detected by the Sloan Degital Sky Survey (SDSS), and the second with the $H_{\mathrm{I}}$ Parkes All-Sky Survey (HIPASS) selected in $21-\mathrm{cm}$. We regard these as the two main samples in this paper, and we can check the dependence on the sample selection using these. In addition, we construct a third sample to extend the dynamic range of the surface density of star formation rate (SFR), which is the cross-match with the Kennicutt (1998, hereafter K98) sample of nearby circumnuclear starburst (SB) galaxies.

\section{SED FITTING}

We mainly use the physical dust model of Draine \& Li (2007, hereafter DL07) to derive physical quantities about dust emission.

The basic parameter of infrared emission in the physical dust model is the heating radiation field strength, $U$, which is the dimensionless energy density of the dust-heating radiation normalized by the local value of the interstellar radiation field (ISRF) around the solar neighbourhood. In reality, the field strength can be different at different locations in a galaxy. However, here we assumed that infrared emission from a galaxy 

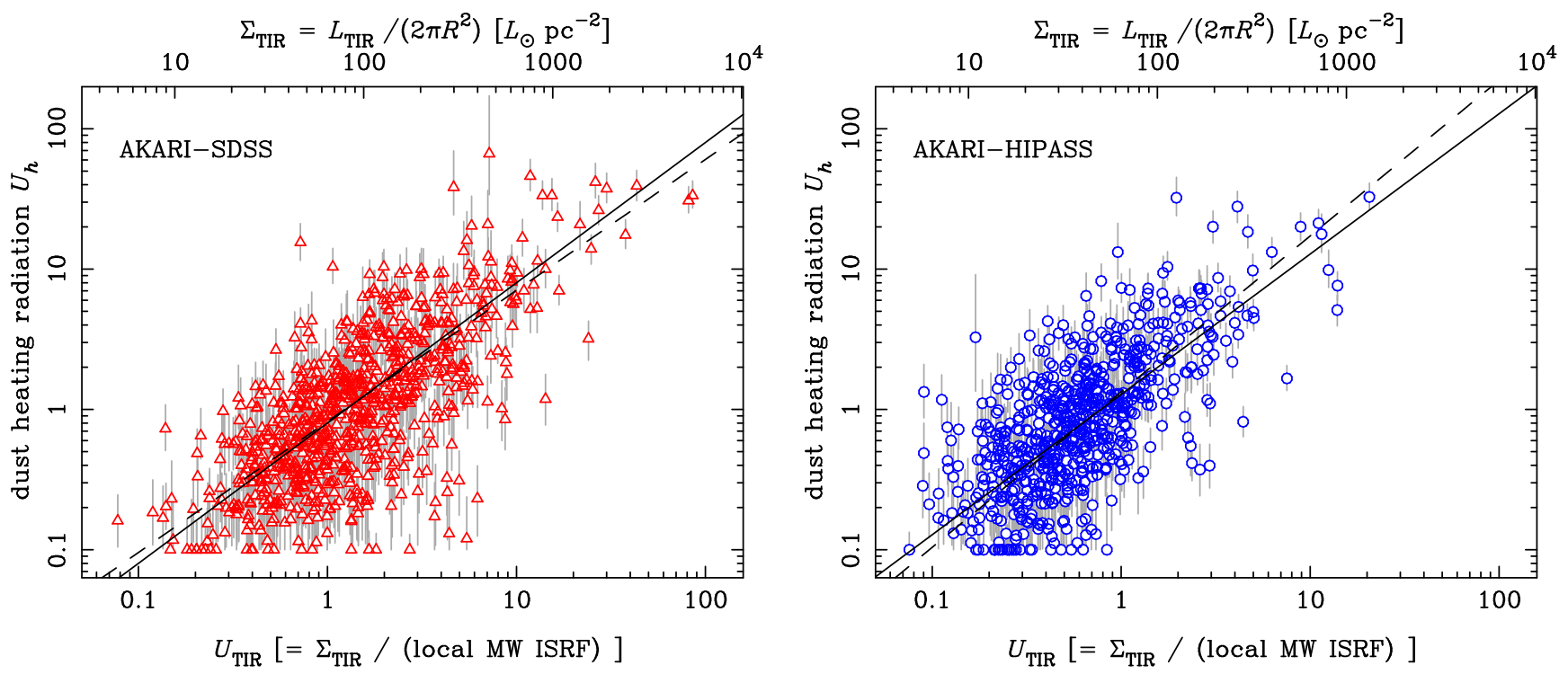

Fig. 2. Dust-heating radiation field strength $\left(U_{h}\right.$, estimated by infrared SED fittings) versus total infrared surface brightness $\left(\Sigma_{\text {TIR }}\right)$, for the AKARI-SDSS (left) and AKARI-HIPASS (right) samples. The parameter $U_{\text {TIR }}$ is $\Sigma_{\text {TIR normalized }}$ by the bolometric strength of the local (solar neighborhood) ISRF in the Milky Way, which can directly be compared with $U_{h}$. The solid and dashed lines are the best-fit linear $\left(U_{h} \propto U_{\mathrm{TIR}}\right)$ and power-law $\left(U_{h} \propto U_{\mathrm{TIR}}^{b}\right)$ relations, respectively.
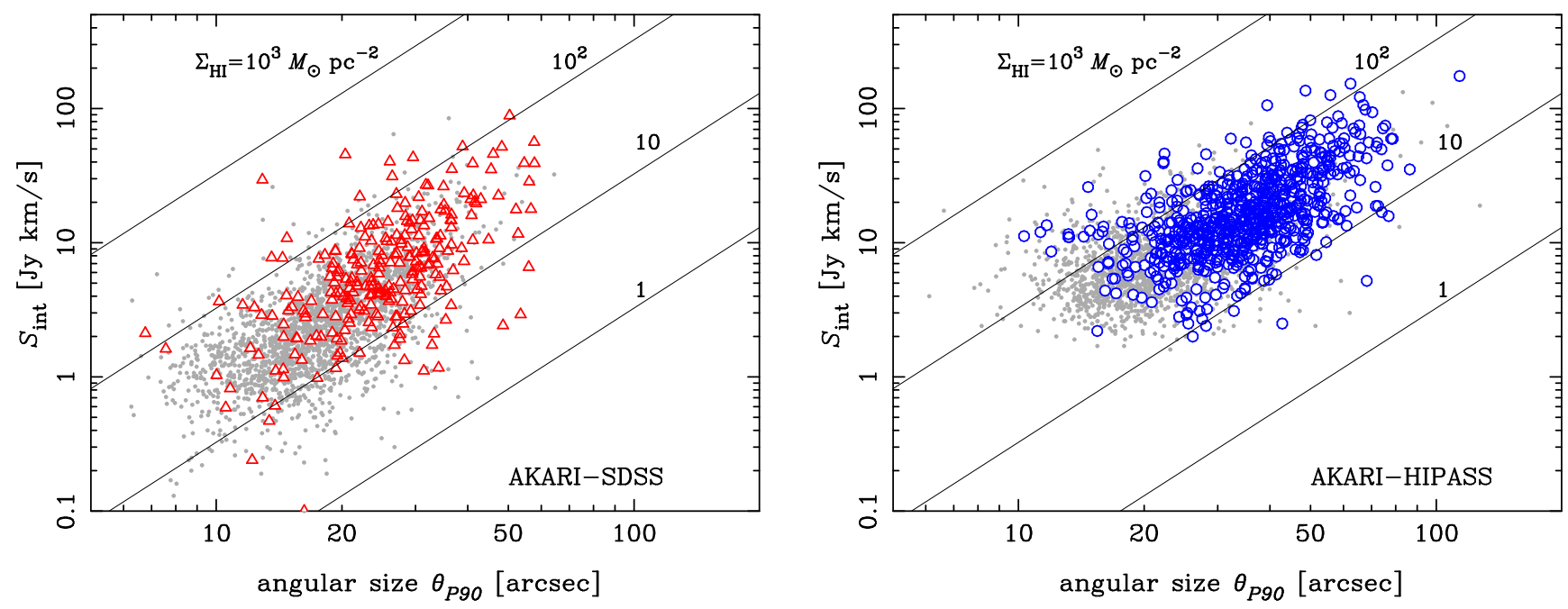

Fig. 3. Velocity-integrated flux density of the $21-\mathrm{cm}$ emission $\left(S_{\mathrm{int}}\right)$ versus angular size $\left(\theta_{P 90}\right)$, for the AKARI-SDSS (left) and AKARI-HIPASS (right) samples. The grey dots are for galaxies whose infrared emission was not detected by AKARI. The solid lines correspond to several values of $\mathrm{H}_{\mathrm{I}}$ column density $\Sigma_{\mathrm{H}_{\mathrm{I}}}$, as indicated in the panels $\left(S_{\text {int }} \propto M_{\mathrm{H}_{\mathrm{I}}} / d_{\mathrm{L}}^{2}\right.$, $\theta_{P 90}=R / d_{\mathrm{A}}$, and hence $\Sigma_{\mathrm{H}_{\mathrm{I}}} \propto S_{\mathrm{int}} / \theta_{P 90}^{2}$, where $d_{\mathrm{L}}$ and $d_{\mathrm{A}}$ are the luminosity and angular diameter distance, respectively). For solid lines we assumed a redshift $z=0.02$ that is typical for the samples here, though the dependence on $z$ is very small, only proportional to the cosmological effect $(1+z)$.

can be described by a single characteristic heating radiation strength, $U_{h}$, for simplicity.

We show examples of the SED fittiings for galaxies in the AKARI-SDSS sample in Fig. 1.

\section{RESULTS}

4.1. Infrared SED versus Global Infrared Radiation Field Strength

Fig. 2 shows the correlation between $U_{h}$ and $U_{\text {TIR }}$, i.e., the total infrared surface brightness $\Sigma_{\mathrm{TIR}}$ normalized 
by the solar neighbourhood value of ISRF. The relation is well described by the linear relation of $U_{h} \propto U_{\mathrm{TIR}}$, with the ratio $U_{h} / U_{\text {TIR }} \sim 1$. This is the tightest correlation of dust temperature ever known, and the dispersion along the mean relation is $13 \%$ in dust temperature

This result strongly indicates that (1) the integrated infrared SED of a galaxy is mostly determined by the general ISRF on the global scale of a galaxy, and (2) dust-heating sources are deeply embedded in an optically-thick dust disk, i.e., the scale height of heating sources being smaller than one optical depth of the dust disk.

\subsection{Radiative Feedback in Optically Thin Galaxies?}

We plot in Fig. 3 the velocity-integrated 21-cm flux density $\left(S_{\text {int }}\right)$ versus angular size $\left(\theta_{P 90}\right)$ for the SDSS$H_{\mathrm{I}}$ and HIPASS samples, in comparison with the AKARI-SDSS and AKARI-HIPASS galaxies. It can be seen that there is a paucity of galaxies in the region of $\Sigma_{\mathrm{H}_{\mathrm{I}}} \lesssim 10 M_{\odot} \mathrm{pc}^{-2}$, roughly corresponding to $\tau_{\text {tot }} \lesssim 1$, where $\tau_{\text {tot }}$ is the effective dust opacity averaged over wavelength.

We consider that there is a physical process reducing the efficiency of galaxy formation when $\tau_{\text {tot }} \lesssim 1$. Because the opacity is with regard to the dust-heating radiation, it is most likely the radiative feedback by the dust photoelectric heating of the ISM. A part (typically $1-10 \%$ ) of the radiation energy absorbed by dust grains is converted to photoelectrons that subsequently heat the ISM. This effect is large enough to be the dominant heating process in many phases of the ISM in galaxies (see, e.g., Bakes \& Tielens, 1994; Wolfire et al., 1995; Rubin et al., 2009). Then, in $\tau_{\text {tot }} \lesssim 1$ galaxies, it is expected that the lack of self-shielding of dust-heating radiation leads to the suppression of star formation activity and reduction of neutral gas in ISM on a galactic scale.

Now we may interpret the overall trend of the galaxy distribution on the $\Sigma_{\mathrm{SFR}}-\Sigma_{\mathrm{H}}$ plane by the strong dependence of SFE on the dust opacity, as follows. There are very few galaxies having $\tau_{\text {tot }} \ll 1$, because the feedback suppresses efficient star formation. Galaxies appear in the region of $\tau_{\text {tot }} \gtrsim 1$ with a large dispersion in the Kennicutt-Schmidt law, because SFE rapidly increases around $\tau_{\text {tot }} \sim 1$. Then SFE continues to increase and asymptotically reaches the maximum value of $\psi_{e, \max } \sim 10 \mathrm{Gyr}^{-1}$ at $\tau_{\text {tot }} \gg 1$. We expect many galaxies around $\tau_{\text {tot }} \sim 1$ because star formation time scales are relatively longer those that in more dusty galaxies.

\section{REFERENCES}

Chanial, P., Flores, H., Guiderdoni, B., Elbaz, D., Hammer, F., \& Vigroux, L., 2007, The Infrared Compactness-Temperature Relation for Quiescent and Starburst Galaxies, A\&A, 462, 81 (C07) Chapin, E. L., Hughes, D. H., \& Aretxaga, I., 2009, The Local Far-Infrared Galaxy ColourLuminosity Distribution: a Reference for BLAST and Herschel/SPIRE Submillimetre Surveys, MNRAS, 393, 653

Chary, R. \& Elbaz, D., 2001, Multicolor Optical Imaging of Infrared-Warm Seyfert Galaxies. III. Surface Photometry: Light Profile Decomposition, ApJ, 556, 562

Devereux, N., 1987, The Spatial Distribution of 10 Micron Luminosity in Spiral Galaxies, ApJ, 323, 91

Draine, B. T. \& Li, A., 2007, Infrared Emission from Interstellar Dust. IV. The Silicate-Graphite-PAH Model in the Post-Spitzer Era, ApJ, 657, 810 (DL07)

Guiderdoni, B., Hivon, E., Bouchet, F. R., \& Maffei, B., 1998, Semi-Analytic Modelling of Galaxy Evolution in the IR/Submm Range, MNRAS, 295, 877

Hauser, M. G. \& Dwek, E., 2001, The Cosmic Infrared Background: Measurements and Implications, ARA\&A, 39, 249

Hwang, H. S., et al., 2010, Evolution of Dust Temperature of Galaxies Through Cosmic Time as Seen by Herschel, MNRAS, 409, 75

Kennicutt, R. C., Jr., 1998, The Global Schmidt Law in Star-forming Galaxies, ApJ, 498, 541 (K98)

Lagache, G., Dole, H., \& Puget, J. -L., 2003, Modelling Infrared Galaxy Evolution Using a Phenomenological Approach, MNRAS, 338, 555

Lehnert, M. D. \& Heckman, T. M., 1996, The Nature of Starburst Galaxies, ApJ, 472, 546 (LH96)

Phillipps, S. \& Disney, M., 1988, The Surface Brightness of Spiral Galaxies. IV - Correlations with Far-Infrared Emission, MNRAS, 231, 359

Rujopakarn, W., Rieke, G. H., Eisenstein, D. J., \& Juneau, S., 2011a, Morphology and Size Differences Between Local and High-redshift Luminous Infrared Galaxies, ApJ, 726, 93 
Rujopakarn, W., Rieke, G. H., Weiner, B. J., et al., 2011b, A Mid-Infrared Indicator for Total Infrared Luminosity and Star Formation Rate of Local and High-Redshift Galaxies, arXiv:1107.2921

Soifer, B. T., Sanders, D. B., Madore, B. F., Neugebauer, G., Danielson, G. E., Elias, J. H., Lonsdale, C. J., \& Rice, W. L., 1987a, The IRAS Bright Galaxy Sample. II - The Sample and Luminosity Function, ApJ, 320, 238

Soifer, B. T. \& Neugebauer, G., 1991, The Properties of Infrared Galaxies in the Local Universe, AJ, 101,354

Takeuchi, T. T., Ishii, T. T., Hirashita, H., Yoshikawa, K., Matsuhara, H., Kawara, K., \& Okuda, H., 2001, Exploring Galaxy Evolution from Infrared Number Counts and Cosmic Infrared Background, PASJ, 53, 37

Totani, T. \& Takeuchi, T. T., 2002, A Bridge from Optical to Infrared Galaxies: Explaining Local Properties and Predicting Galaxy Counts and the Cosmic Background Radiation, ApJ, 570, 470

Totani, T., Takeuchi, T. T., Nagashima, M., Kobayashi, M. A. R., \& Makiya, R., 2011, Infrared Spectral Energy Distribution of Galaxies in the AKARI All Sky Survey: Correlations with Galaxy Properties, and Their Physical Origin, PASJ, 63, 1181

Valiante, E., Lutz, D., Sturm, E., Genzel, R., \& Chapin, E. L., 2009, A Backward Evolution Model for Infrared Surveys: The Role of AGNand Color- $\mathrm{L}_{T I R}$ Distributions, ApJ, 701, 1814

Walcher, J., Groves, B., Budavári, T., \& Dale, D., 2011, Fitting the Integrated Spectral Energy Distributions of Galaxies, Ap\&SS, 331, 1 